\title{
The Tolman-Bondi-Vaidya Spacetime: matching timelike dust to null dust
}

\author{
Sijie Gad* and José P. S. Lemo: \\ Centro Multidisciplinar de Astrofísica - CENTRA, \\ Departamento de Física, Instituto Superior Técnico, \\ Universidade Técnica de Lisboa, \\ Av. Rovisco Pais 1, 1049-001 Lisboa, Portugal
}

\begin{abstract}
The Tolman-Bondi and Vaidya solutions are two solutions to Einstein equations which describe dust particles and null fluid, respectively. We show that it is possible to match the two solutions in one single spacetime, the Tolman-Bondi-Vaidya spacetime. The new spacetime is divided by a null surface with Tolman-Bondi dust on one side and Vaidya fluid on the other side. The differentiability of the spacetime is discussed. By constructing a specific solution, we show that the metric across the null surface can be at least $C^{1}$ and the stress-energy tensor is continuous.
\end{abstract}

PACS numbers: 04.20.-q, 04.40.Nr

\section{INTRODUCTION}

The Tolman-Bondi solution describes the inhomogeneous gravitational collapse of spherical dust. In comoving coordinates, the metric is given by 1, 2]

$$
d s^{2}=-d t^{2}+\frac{r^{\prime 2}}{1+E(R)} d R^{2}+r^{2} d \Omega^{2},
$$

where $d \Omega^{2}$ is the metric of the unit two-sphere, $E(R)$ is an arbitrary function of the comoving radial coordinate $R$, and the prime denotes the partial derivative with respect to $R$. The function $r(t, R)$ is a solution of

$$
\dot{r}^{2}=\frac{2 M(R)}{r}+E(R),
$$

where the overdot denotes the partial derivative with respect to $t$ and $M(R)$ is the effective gravitational mass within $R$. In contrast, the incoming Vaidya metric describing collapsing null dust is given by [3, 4]

$$
d s^{2}=-\left(1-\frac{2 M(v)}{r}\right) d v^{2}+2 d v d r+r^{2} d \Omega^{2}
$$

where $v$ is an advanced time and the $v=$ constant line gives the trajectory of the null fluid. Both the Tolman and the Vaidya spacetimes provide important examples for the study of naked singularities formed in gravitational collapse. The intrinsic relation between the two solutions had not been investigated for many years. Lemos first showed in [5], that the Vaidya metric (3) can be obtained from the Tolman-Bondi metric (11) by taking the limit $E \rightarrow \infty$. Therefore, the two metrics belong to one family and their naked singularities are of the same nature. Hellaby [6] pointed out that Lemos' derivation requires that $E$ be a constant through the spacetime. As a generalization, Hellaby showed that this assumption can

\footnotetext{
*Electronic address: sijie@fisica.ist.utl.pt
}

${ }^{\dagger}$ Electronic address: lemos@fisica.ist.utl.pt be dropped and the same Vaidya limit can be achieved from the Tolman-Bondi solutions by using a different coordinate transformation.

Despite the connection discovered above, the two solutions have been treated in two separate spacetimes which are connected only mathematically by taking the limit on $E$. Is it possible to put the two solutions in one single spacetime such that the Tolman-Bondi dust can approach the Vaidya fluid as a physical limit? Hellaby's work has provided some necessary mathematical framework to solve this problem. Hellaby makes no assumptions on the function $E(R)$ in the Tolman-Bondi model. This is an important point for our generalization. In [6], the Vaidya limit is obtained by taking the limit $E(R) \rightarrow \infty$ everywhere.

Our approach to matching the two solutions in one spacetime is simple. Instead of requiring $E(R) \rightarrow \infty$ everywhere, we require $E(R)$ to diverge only at one hypersurface $R=R_{0}$. For $R<R_{0}, E(R)$ is finite and it corresponds to a Tolman-Bondi solution. For $R>R_{0}$, the spacetime transfers into the Vaidya null fluid. In this new spacetime, dust particles which follow timelike geodesics and photons which follow null geodesics coexist and a phase transition occurs across the $R=R_{0}$ hypersurface. By imposing some general conditions, the spacetime is continuous $\left(C^{0}\right)$ by construction. An important issue to be discussed is the smoothness around the limit surface $R=R_{0}$, i.e., how well one can match the two solutions together. We show from a specific example, that the metric across the limit surface can be at least $C^{1}$. We also show that the stress-energy tensor and the Kretschmann scalar are both continuous in the spacetime.

\section{CONSTRUCTION OF THE SPACETIME}

In this section, we shall construct a spherically symmetric spacetime which combines the Tolman-Bondi dust and the Vaidya null dust. To be definitive, the TolmanBondi dust will be configured inside the Vaidya dust. However, all of our arguments and results apply to the reversed configuration. We start with the Tolman- 
Bondi metric (11). It is evident from this metric that each comoving observer follows a timelike worldline with $R=$ constant. To approach the Vaidya limit, we shall let $E(R)$ diverge at some fixed $R=R_{0}>0$ such that the spacetime consists of the Tolman-Bondi dust for $R<R_{0}$ and the Vaidya dust for $R \geq R_{0}$. If this can be done, the $R=R_{0}$ surface will become a limit null surface for Tolman-Bondi observers, which indicates that the original Tolman-Bondi coordinates in Eq. (1) will break down at $R=R_{0}$. This is reminiscent of the Schwarzschild solution where the original Schwarzschild coordinates break down when one approaches the event horizon, and the introduction of the Kruskal extension is necessary to eliminate the apparent coordinate singularity. Now we face a similar situation and we shall introduce a new set of coordinates to extend the spacetime from the Tolman-Bondi interior to the Vaidya exterior, to yield a Tolman-BondiVaidya spacetime. Following [6], we rewrite Eq. (10), eliminating $d t$ through

$$
d r=\dot{r} d t+r^{\prime} d R \rightarrow d t=\frac{-r^{\prime} d R+d r}{\dot{r}}
$$

and then using Eq. (2), to obtain

$$
\begin{aligned}
d s^{2}= & -\left(1-\frac{2 M}{r}\right) \frac{r^{2}}{[1+E(R)] \dot{r}^{2}} d R^{2}+2 \frac{r^{\prime}}{\dot{r}^{2}} d R d r \\
& -\frac{1}{\dot{r}^{2}} d r^{2}+r^{2} d \Omega^{2}
\end{aligned}
$$

Now the metric is expressed in terms of $(R, r, \theta, \phi)$. Since $r$ is the areal radius, it should be kept as a good coordinate in the extended spacetime. We note, in passing, that Hellaby further introduced a new coordinate $v$ defined by $v=\int_{0}^{R} \frac{a^{\prime}}{\sqrt{E}} d R-\frac{r}{2 E}$, where $a$ is some function of $R$, such that the metric is finally expressed in coordinates $(v, r, \theta, \phi)$. However, the components of the metric involve functions like $E(R)$ and $M(R)$ that are implicit functions of $(v, r)$. Consequently, it will be difficult to study the differentiability of the metric with Hellaby's coordinate system. Indeed we find that introducing the new coordinate $v$ is not necessary here. Under certain conditions, coordinates $(R, r, \theta, \phi)$ are good enough for our purposes to describe the spacetime. To proceed, we first give some limiting forms of the components of the metric (5) which has been derived by Hellaby in [6].

The exact solution for $r^{\prime}$ in a collapsing model is [8]

$$
r^{\prime}=\left(\frac{M^{\prime}}{M}-\frac{E^{\prime}}{E}\right) r-\left[a^{\prime}-\left(\frac{M^{\prime}}{M}-\frac{3 E^{\prime}}{2 E}\right)(a-t)\right] \dot{r},(6)
$$

where $a=a(R)$ is another arbitrary function. We shall show in section 11 B that $t$ can be written as an explicit function of $(R, r)$. Thus, each component in the metric of Eq. (5) depends on $(R, r, \theta, \phi)$. Hellaby required that $M$ and $r$ be finite while $E$ goes to infinity everywhere. Here we adopt a similar requirement with only a small modification, albeit important: we require $M$ and $r$ remain finite everywhere and $E(R)$ is divergent for $R \geq R_{0}$. Then we have the following limiting forms at $R=R_{0}[\underline{6}]$ :

$$
\begin{aligned}
r & \rightarrow \sqrt{E}(a-t) \\
\dot{r} & \rightarrow-\sqrt{E} \\
r^{\prime} & \rightarrow \frac{r E^{\prime}}{2 E}+a^{\prime} \sqrt{E}
\end{aligned}
$$

By simple substitution, we find the following limits for the components of Eq. (5) (see []ㅁ)

$$
\begin{aligned}
\frac{1}{\dot{r}^{2}} & \rightarrow \frac{1}{E} \\
\frac{r^{\prime}}{\dot{r}^{2}} & \rightarrow\left(\frac{a^{\prime}}{\sqrt{E}}+\frac{r E^{\prime}}{2 E^{2}}\right), \\
\frac{r^{\prime 2}}{(1+E) \dot{r}^{2}} & \rightarrow\left(\frac{E}{1+E}\right)\left(\frac{a^{\prime}}{\sqrt{E}}+\frac{r E^{\prime}}{2 E^{2}}\right)^{2} .
\end{aligned}
$$

If we further impose restrictions such that

$$
\frac{a^{\prime}}{\sqrt{E}}+\frac{r E^{\prime}}{2 E^{2}} \rightarrow 1 \quad \text { at } \quad R=R_{0}
$$

and identical to 1 for $R>R_{0}$, one sees immediately, by comparing the components of the original TolmanBondi metric (5) and those of the Vaidya metric (3) and identifying $v$ with $R$, that the metric of Eq. (5) becomes exactly the Vaidya metric for $R>R_{0}$. In summary, our spacetime is constructed in the following way: The coordinates are $(R, r, \theta, \phi)$. For $R<R_{0}$, the spacetime is described by any Tolman-Bondi dust in the form of (5) which satisfies the limiting condition (9) and $E \rightarrow \infty$ at $R=R_{0}$. For $R>R_{0}$, the spacetime is described by the Vaidya null dust (3) with $v$ relabeled by $R$. In this case, we can choose a smooth mass function $M(R)$ for the entire spacetime. With this choice, the spacetime metric is continuous (or $C^{0}$ ) by construction. For future reference, we write the complete metric as follows

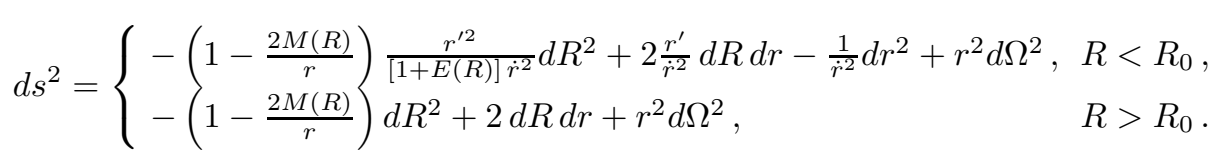


nate of the Vaidya null dust. This shows the unification of the two solutions expressed in this coordinate system. It is also easy to check that the $R R$ component of the inverse metric, $g^{R R}$, approaches zero as $R \rightarrow R_{0}$, which confirms that the hypersurface $R=R_{0}$ is a null hypersurface. In the next section, we shall show through a concrete example that the metric at $R=R_{0}$ can be at least $C^{1}$.

\section{AN EXAMPLE}

To demonstrate how well the two metrics can be matched, we provide an explicit example based on the prescription given in the previous section.

\section{A. Choosing the arbitrary functions}

Since our interest is matching the Tolman-Bondi and Vaidya solutions, we shall restrict our attention to the neighborhood of the limit null hypersurface, which is denoted by $\Sigma$. Since at $R=R_{0}$ the function $E(R)$ goes to infinity, it is convenient to discuss the smoothness of $E(R)$ at $R=R_{0}$ through an auxiliary function $h(R)$ given by

$$
h(R)=\frac{1}{E(R)}
$$

According to the discussion in the previous section, $h(R)$ should approach zero at $\Sigma$ from the Tolman-Bondi inte- rior $\left(R<R_{0}\right)$ and be identical zero in the Vaidya exterior. Hence, we choose $h(R)$ as

$$
h(R)= \begin{cases}\left(R_{0}-R\right)^{3} & R<R_{0} \\ 0 & R \geq R_{0}\end{cases}
$$

To satisfy the limit (9), we define $a(R)$ in the TolmanBondi interior as

$$
a(R)=\frac{2}{\sqrt{R_{0}-R}}
$$

In the following two subsections, we shall investigate the differentiability of the metric associated with the model spacetime and the continuity of the Einstein tensor across the null surface $\Sigma$.

\section{B. Differentiability of the metric}

Our task in this subsection is to show that the metric is $C^{1}$ on the null surface $\Sigma$. We shall show that the components of the metric is differentiable on $\Sigma$.

We begin with the $R R$ component in Eq. (10), denoting it on the Tolman-Bondi side, $R<R_{0}$, by $g_{R R}^{-}$, and on the Vaidya side, $R>R_{0}$, by $g_{R R}^{+}$. Combining Eqs. (2), (15), (11), (12) and (13), one may write $g_{R R}^{-}$as a function of $(R, r)$

$$
\begin{aligned}
g_{R R}^{-}= & \frac{-1+\frac{2 M(R)}{r}}{\left(1+\frac{1}{\left(R_{0}-R\right)^{3}}\right)\left(\frac{1}{\left(R_{0}-R\right)^{3}}+\frac{2 M(R)}{r}\right)} \times\left[r\left(\frac{3}{R-R_{0}}+\frac{M^{\prime}(R)}{M(R)}\right)+\sqrt{\frac{1}{\left(R_{0}-R\right)^{3}}+\frac{2 M(R)}{r}}\right. \\
& \left.\left(\frac{1}{\left(R_{0}-R\right)^{3 / 2}}-\left(\frac{2}{\sqrt{R_{0}-R}}-t(R, r)\right)\left(\frac{9}{2 R-2 R_{0}}+\frac{M^{\prime}(R)}{M(R)}\right)\right)\right]^{2},
\end{aligned}
$$

where we have expressed $t$ formally as a function of $(R, r)$. To find the explicit form of $t(R, r)$, we consider the hyperbolic solution for the collapsing model $[6]$

$$
\begin{aligned}
r & =\frac{M}{E}(\cosh \eta-1), \\
(\sinh \eta-\eta) & =\frac{E^{3 / 2}(a-t)}{M}
\end{aligned}
$$

Solving Eq. (15) for $\eta$ gives

$$
\eta=\cosh ^{-1}\left(1+\frac{E r}{M}\right)
$$

where $\cosh ^{-1}$ is the inverse function of cosh and is required to be positive. By symmetry, there is also a neg- ative solution for $\eta$. But one can easily check that, as $E \rightarrow \infty$, the negative $\eta$ leads to $r \rightarrow \sqrt{f}(t-a)$, which is not a collapsing solution. Therefore $\eta$ is large and positive in the region of interest, which implies that $\sinh \eta$ is positive too. Thus, from Eqs. (15) and (16), we find

$$
t=a-\frac{\sqrt{E r(2 M+E r)}-M \cosh ^{-1}(1+E r / M)}{E^{3 / 2}} .
$$

It is obvious that $t$ diverges at $R=R_{0}$ for the functions chosen in section IIIA This confirms the breakdown of the original coordinates, as we have mentioned at the beginning of section $\amalg$ Now we have $g_{R R}^{-}$as an explicit function of $(R, r)$. Its derivatives can then be calculated straightforwardly. To obtain the limits of these deriva- 
tives at $R=R_{0}$, we treat $R_{0}-R$ as a small quantity and compute the power series expansion about $R=R_{0}$. This treatment guarantees the accuracy of the final results because no approximation has to be made in the intermediate steps. With the help of Mathematica, the results can be obtained almost instantly. We find that at $R=R_{0}$

$$
\begin{aligned}
& \frac{\partial g_{R R}^{-}}{\partial r}=\frac{\partial g_{R R}^{+}}{\partial r}=-\frac{2 M\left(R_{0}\right)}{r^{2}} \\
& \frac{\partial g_{R R}^{-}}{\partial R}=\frac{\partial g_{R R}^{+}}{\partial R}=\frac{2 M^{\prime}\left(R_{0}\right)}{r}
\end{aligned}
$$

Therefore, the $R R$ component of the metric is differentiable (or $C^{1}$ ) at $\Sigma$.

With similar notations, we repeat the procedure above for $g_{R r}^{-}$and $g_{r r}^{-}$and find that their derivatives with respect to $R$ and $r$ vanish as $R \rightarrow R_{0}$, agreeing with their Vaidya counterparts (note that $g_{R r}^{+}$and $g_{r r}^{+}$are both constants). Therefore, we have shown that the spacetime metric is $C^{1}$ across the null hypersurface $\Sigma$.

However, it is worth mention, as a remark, that there are examples where the metric is not always $C^{1}$ on $\Sigma$ when the conditions in section III are satisfied. Here is a counterexample. We choose, in the $R<R_{0}$ region, $h(R)=\left(R_{0}-R\right)^{2}$ and $a(R)=-\ln \left(R_{0}-R\right)$. It is easy to check that the limiting condition (9) is satisfied for this choice. By repeating the similar calculation we carried out for the first example, we find that $\partial g_{R r}^{-} / \partial R \rightarrow-r$ when $R \rightarrow R_{0}$, which is not equal to the $g_{R r}^{+} / \partial R \rightarrow 0$ limit except for the $r=0$ singularity. Thus, this example shows that the metric is not differentiable (or $C^{1}$ ) on $\Sigma$. There is a qualitative explanation for why the first example works while the second one does not. Since $h(R)$ vanishes identically for $R>R_{0}$, we see $h(R)$ defined by Eq. (12) is $C^{2}$ at $R=R_{0}$ while $h(R)$ defined above is just $C^{1}$. Roughly speaking, a better behaved function corresponds to a better behaved metric.

\section{Continuity of the stress-energy tensor and the Kretschmann scalar}

We have shown that the spacetime metric defined at the beginning of section $\Pi$ is at least $C^{1}$. In this subsection, we shall show that the spacetime performs even better than $C^{1}$. One important quantity associated with the second-order derivatives of the metric is the stressenergy tensor $T_{a b}$, which can be derived from Einstein's equation

$$
G_{a b} \equiv R_{a b}-\frac{1}{2} R g_{a b}=8 \pi T_{a b}
$$

We now calculate $G_{a b}$ associated with the metric defined by Eq. (10) and functions chosen in section ஹI From Einstein's equation, it is straightforward to check that the only nonzero component of $G_{a b}$ for the Vaidya metric is

$$
G_{R R}=\frac{2 M^{\prime}(R)}{r^{2}} .
$$

We need to show that $G_{a b}$ associated with the TolmanBondi metric agrees with this limit at $R=R_{0}$. The calculation follows the same strategy as in subsection IIIB The limits are taken only in the final step by means of power expansion. We finally find that the TolmanBondi stress-energy tensor approaches the Vaidya limit at $R=R_{0}$, i.e., all components of $G_{a b}$ vanish at $R=R_{0}$ except $G_{R R} \rightarrow 2 M^{\prime}\left(R_{0}\right) / r^{2}$.

Another quantity related to the spacetime curvature is the Kretschmann scalar $K=R^{a b c d} R_{a b c d}$, which is given in [6] for the Tolman-Bondi metric

$$
K^{T}=\frac{48 M^{2}}{r^{6}}-\frac{32 M M^{\prime}}{r^{5} r^{\prime}}+\frac{12 M^{\prime 2}}{r^{4} r^{\prime 2}}
$$

and for the Vaidya metric

$$
K^{V}=\frac{48 M^{2}}{r^{6}}
$$

Since $r^{\prime} \rightarrow \infty$ in our model spacetime, we see that the Kretschmann scalar is continuous across $\Sigma$.

\section{CONCLUDING REMARKS}

We have constructed, by generalizing Lemos and Hellaby's methods [5, 6], a spacetime where the TolmanBondi dust and the Vaidya null fluid coexist in harmony. The spacetime is described by a uniform coordinate system which is comoving with both the Tolman-Bondi and Vaidya observers. We have shown, from an explicit example, that the spacetime metric is at least $C^{1}$ across the null surface $\Sigma$. Moreover, we have shown that the stress-energy tensor and the Kretschmann are continuous on $\Sigma$. This property fails for some other spacetimes. For instance, when matching the Friedmann interior to the Schwarzschild exterior [7], the energy density across the matching surface is obviously discontinuous since the Friedmann interior has a uniform distribution of density and the Schwarzschild exterior has a vanishing density. Although the Tolman-Bondi and Vaidya models are two well-known solutions to Einstein's equations, the match of the two models is a new issue. We have studied the possibility of matching them in one spacetime. It remains unknown to us how this match can be realized in a physical process, or what physical mechanism can make it happen.

\section{Acknowledgments}

This work was partially funded by Fundação para a Ciência e Tecnologia (FCT) through project PDCT/FP/50202/2003. SG acknowledges financial support from FCT through grant SFRH/BPD/10078/2002. JPSL thanks Observatório Nacional do Rio de Janeiro for hospitality. 
[1] R. C. Tolman, Proc. Natl. Acad. Sci.l 20, 169 (1934).

[2] J. P. S. Lemos, Phys. Lett. A. 158, 279 (1991).

[3] P. C. Vaidya, Proc. Indian Acad. Sci. A33, 264 (1951).

[4] W. A. Hiscock, L.G. Williams and D.M. Eardley, Phys. Rev. D. 26, 751 (1982).

[5] J. P. S. Lemos, Phys. Rev. Lett. 68, 1447 (1992).
[6] C. Hellaby, Phys. Rev. D 49, 6484 (1994).

[7] C.W. Misner, K.S. Thorne and J.A. Wheeler, Gravitation, W.H.Freemand and Company, New York (1995).

[8] In Eq.(10) of 6], the sign before the bracket "[" is positive. We believe it is a misprint and have corrected it below. 